\title{
Scattering of Obliquely Incident Waves by an Impedance Cylinder with Inhomogeneous Bianisotropic Coating
}

\author{
Bernhard Jakoby, Member, IEEE
}

\begin{abstract}
A propagator matrix approach for cylindrical structures is applied to solve scattering problems featuring inhomogeneous bianisotropic media. The involved propagator matrix, which describes the relation between the transverse fields at the two boundaries of an inhomogeneous cylindrical layer, is obtained by utilizing Fourier series expansions for fields as well as material parameter functions. The presented formalism is capable of analyzing the scattering of plane waves which are obliquely incident on an impedance cylinder that is covered with an inhomogeneous bianisotropic layer. We provide typical examples showing the applicability of the method.
\end{abstract}

Index Terms-Electromagnetic scattering.

\section{INTRODUCTION}

$\mathbf{I}$ $\mathrm{N}$ this contribution we present an approach for the calculation of electromagnetic scattering by an impedance cylinder (characterized by impedance boundary conditions) that features an inhomogeneous bianisotropic coating. The considered type of inhomogeneity includes lateral, i.e., angular, as well as radial inhomogeneities of the bianisotropic coating.

In the last few years, known numerical approaches for isotropic media problems have been generalized and modified to include bi-isotropic (chiral) and special types of bianisotropic media as well. Special interest has been devoted to the scattering by cylindrical objects, see, e.g., [1]-[6]. In [1] scattering from multilayered chiral cylinders with a center conductor is considered, in [2] scattering by a chiral cylinder embedded in another chiral medium is investigated, and in [3] oblique scattering by cylindrically stratified chiral media is treated using a chain matrix method. Obliquely incident waves are also considered in [4], where scattering by a homogeneous bi-isotropic cylinder is investigated, and [5] deals with conductors which are eccentrically coated with chiroplasma cylinders. The backscattering from curved metallic surfaces coated with chiral layers has been considered in [6]. The coating of scatterers with chiral media gained attention due to the additional degree of freedom in the material parameters, which allows to influence the scattering behavior.

Manuscript received July 3, 1995; revised September 11, 1996. This work was supported by Erwin Schrödinger Grant J00975-TEC from the Austrian Fonds zur Förderung der wissenschaftlichen Forschung.

The author is with Delft University of Technology, DIMES, Electronic Instrumentation Laboratory, Delft 2628 CD, The Netherlands.

Publisher Item Identifier S 0018-926X(97)02500-3.
Further references on known numerical and analytical approaches dealing with wave propagation and scattering in chiral media can be found in the above cited papers, as well as in monographs on this topic (e.g., [7], [8]).

Here, we will consider general bianisotropic media, which introduces further degrees of freedom and contains simpler media as special cases. Apart from the coverage of general bianisotropic media, the emphasis lies on the consideration of inhomogeneities in the angular direction of the cylindrical structure, which still is a challenging problem even for simpler media, while inhomogeneities in radial direction have been thoroughly treated in the form of stratified structures. In this paper, we adopt a method similar to one that we successfully used for guided wave problems related to these kind of structures [9]. By employing a spectral expansion of fields and material parameter functions in angular direction, a propagator matrix relating the transverse field components at the bounding surfaces of an inhomogeneous cylindrical layer can be constructed. In principle, this propagator matrix is infinite dimensional but can be truncated for numerical evaluations. By means of this propagator matrix the boundary conditions at the center conductor can be transformed to the layer-free space interface, which allows the determination of the scattered fields due to an incident plane wave.

\section{THEORY}

We assume harmonic time-dependence $\exp (j \omega t)$ and constitutive equations in the Tellegen form [10]

$$
\begin{aligned}
& \mathbf{D}=\underline{\underline{\varepsilon}} \cdot \mathbf{E}+\underline{\underline{\xi}} \cdot \mathbf{H} \\
& \mathbf{B}=\underline{\underline{\zeta}} \cdot \mathbf{E}+\underline{\underline{\mu}} \cdot \mathbf{H}
\end{aligned}
$$

where the material parameter tensors $\underline{\underline{\varepsilon}}, \underline{\mu}, \underline{\xi}$, and $\underline{\underline{\zeta}}$ describe the bianisotropic material behavior at frequency $\bar{\omega}$. In the following we will frequently use cylindrical coordinates $r, \phi, z$ with $x=r \cos \phi, y=r \sin \phi$. We consider structures as shown in Fig. 1. The impedance core filling up the region $r<r_{1}$ is covered by a layer of thickness $r_{2}-r_{1}$. This layer is inhomogeneous where the material parameters are functions of the lateral position described by $\phi$ and $r$. A plane wave is obliquely incident in some direction characterized by angles $\theta_{0}$ and $\phi_{0}$ (see Fig. 1). The exponential associated with the incident wave is given by $\exp \left(-j \mathrm{k}_{0} \cdot \mathrm{r}\right)$, where $\mathbf{k}_{0}=k_{0}\left(\hat{\mathbf{x}} \sin \theta_{0} \cos \phi_{0}+\hat{\mathbf{y}} \sin \theta_{0} \sin \phi_{0}+\hat{\mathbf{z}} \cos \theta_{0}\right)$ and $k_{0}=\omega \sqrt{\mu_{0} \varepsilon_{0}}$. As the structure is infinitely extended 


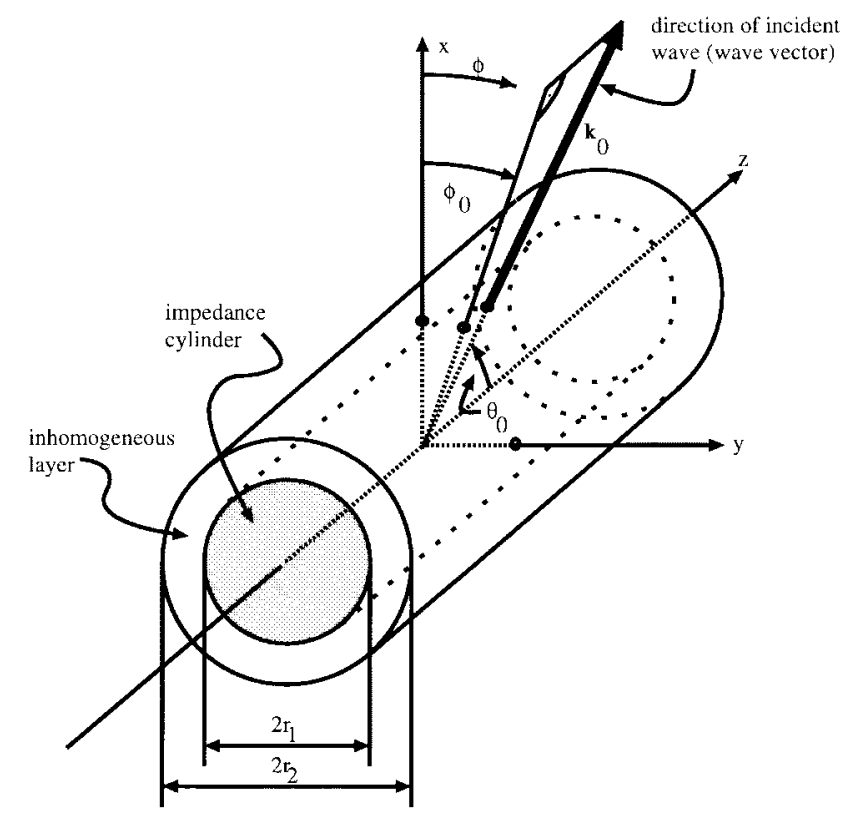

Fig. 1. Principal geometry and direction of incident wave.

in $z$ direction the scattered fields must obey the $z$-dependence $\exp \left(-j k_{0} \cos \theta_{0} z\right)$.

\section{A. Determination of the Propagator Matrix}

In this section, we will give a brief outline of the derivation of the propagator matrix for the inhomogeneous layer without repeating various details which are given in our previous work [9]. Writing Maxwell's equations for a general bianisotropic medium in cylindrical coordinates, the $r$ components of the field vectors can be eliminated to arrive at

$$
\underline{\underline{L}} \cdot \mathbf{f}=\frac{\partial}{\partial r} \mathbf{f}
$$

with $\mathbf{f}=\left[E_{\phi}, E_{z}, H_{\phi}, H_{z}\right]^{T}$ (the superscript $T$ denotes transposition) and

$$
\underline{\underline{L}}=\underline{\underline{\mathcal{L}}}\left(r, \frac{1}{r} \frac{\partial}{\partial \phi}, \frac{\partial}{\partial z}, \varepsilon_{k l}, \mu_{k l}, \xi_{k l}, \zeta_{k l l}\right)
$$

where $\varepsilon_{k l}, \mu_{k l}, \xi_{k l}$, and $\zeta_{k l}$ stand for the $r, \phi$ dependent elements of the material parameter tensors in cylindrical coordinates $(k, l=r, \phi, z)$. The explicit form of the operator matrix $\underline{\mathcal{L}}$ is given in Appendix A (see also [9]).

Due to the known form of the $z$ dependence for the fields, derivatives with respect to $z$ can simply be replaced by $-j k_{z}=-j k_{0} \cos \theta_{0}$. Next, by considering that field components and material parameters must be $2 \pi$ periodic with respect to $\phi$, we can expand these quantities as Fourier series

$$
\begin{aligned}
\psi(r, \phi, z) & =e^{-j k_{z} z} \sum_{n=-\infty}^{\infty} \tilde{\psi}_{n}\left(r, k_{z}\right) e^{-j n \phi} \\
g(r, \phi) & =\sum_{n=-\infty}^{\infty} \tilde{g}_{n}(r) e^{-j n \phi}
\end{aligned}
$$

where $\psi$ stands for any transverse field component and $g$ denotes any of the scalar functions occurring in $\underline{\underline{L}}$, which depend upon material parameters only. Substitution of the expansions (5) into (3) leads to an infinite dimensional matrix equation in terms of the spectral field components [9]

$$
\tilde{\underline{\mathbf{L}}} \cdot \tilde{\mathbf{f}}=\frac{\partial}{\partial r} \tilde{\mathbf{f}}
$$

where $\tilde{\mathbf{f}}=\left[\overrightarrow{\tilde{E}}_{\phi}, \overrightarrow{\tilde{E}}_{z}, \overrightarrow{\tilde{H}}_{\phi}, \overrightarrow{\tilde{H}}_{z}\right]^{T}$ and $\overrightarrow{\tilde{E}}_{\phi}=\left[\ldots, \tilde{E}_{\phi,-1}, \tilde{E}_{\phi, 0}\right.$, $\left.\tilde{E}_{\phi, 1}, \ldots\right]^{T}$ denotes an infinite dimensional vector containing the Fourier series coefficients $\tilde{E}_{\phi, n}(n=-\infty, \ldots, \infty)$ of $E_{\phi}$ and similarly for $\overrightarrow{\tilde{E}}_{z}, \overrightarrow{\tilde{H}}_{\phi}$, and $\overrightarrow{\tilde{H}}_{z}$. Note that these vectors must not be confused with the "physical" field vectors (printed in bold face). For numerical treatment, these infinite dimensional vectors have to be truncated by setting their Fourier coefficients with index $|n|>N$ to zero. The effects of the truncation and numerical convergence, with respect to the truncation parameter $N$, were discussed in [9].

Integration of the differential equation (6) yields the prop-

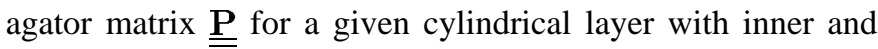
outer radius $r_{1}$ and $r_{2}$, respectively

$$
\tilde{\mathrm{f}}\left(r_{2}\right)=\underline{\underline{\mathbf{P}}}\left(r_{2}, r_{1}\right) \cdot \tilde{\mathrm{f}}\left(r_{1}\right) \text {. }
$$

The integration of (6) can be performed numerically by subdividing the layer into $L$ sublayers being so thin that $\underline{\underline{\mathbf{\mathbf { L }}}}$ is assumed to be constant with respect to $r$ within each sublayer

$$
\underline{\underline{\mathbf{P}}}\left(r_{2}, r_{1}\right) \approx \prod_{n=L-1}^{0}\left[\underline{\underline{\mathbf{I}}}+\underline{\underline{\mathbf{\mathbf { L }}}}\left(r_{1}+n \Delta r\right) \Delta r\right] .
$$

Here, $\underline{\underline{\mathbf{I}}}$ denotes the unit matrix and $\Delta r=r_{2}-r_{1} / L$ is the thickness of a sublayer.

\section{B. Calculation of the Scattered Fields}

A relation between the transverse components of the scattered field solutions in free space can be obtained by employing a common solution Ansatz where the $z$-components of the scattered spectral fields are set proportional to $H_{n}^{(2)}\left(k_{r} r\right)$. Here, $k_{r}=k_{0} \sin \theta_{0}$ is the radial wavenumber and $H_{n}^{(2)}$ denotes Hankel's function of second kind and order $n$, which was chosen to fulfill the radiation condition at infinity. The order $n$ corresponds to the $n$th Fourier coefficients of the fields, i.e., $\tilde{E}_{z, n}^{s}$ and $\tilde{H}_{z, n}^{s}$ (the superscripts $s$ indicate the scattered fields). Using Maxwell's equations, the spectral $\phi$ components at $r=r_{2}$ can then be expressed in terms of the spectral $z$ components

$$
\left[\begin{array}{l}
\tilde{E}_{\phi, n}^{s}\left(r_{2}\right) \\
\tilde{H}_{\phi, n}^{s}\left(r_{2}\right)
\end{array}\right]=\underline{\underline{\mathbf{F}}}_{n} \cdot\left[\begin{array}{l}
\tilde{E}_{z, n}^{s}\left(r_{2}\right) \\
\tilde{H}_{z, n}^{s}\left(r_{2}\right)
\end{array}\right]
$$

where

$$
\begin{aligned}
\underline{\underline{\mathbf{F}}}_{n} & =\left[\begin{array}{ll}
F_{11, n} & F_{12, n} \\
F_{21, n} & F_{22, n}
\end{array}\right] \\
& =\frac{1}{k_{r}^{2}}\left[\begin{array}{cc}
-\frac{n k_{0} \cos \theta_{0}}{r_{2}} & j \omega \mu_{0} k_{r} \frac{H_{n}^{(2) \prime}\left(k_{r} r_{2}\right)}{H_{n}^{(2)}\left(k_{r} r_{2}\right)} \\
-j \omega \varepsilon_{0} k_{r} \frac{H_{n}^{(2) \prime}\left(k_{r} r_{2}\right)}{H_{n}^{(2)}\left(k_{r} r_{2}\right)} & -\frac{n k_{0} \cos \theta_{0}}{r_{2}}
\end{array}\right] .
\end{aligned}
$$

Here, $H_{n}^{(2) \prime}$ denotes the derivative of $H_{n}^{(2)}$ with respect to its argument.

Next, we express the obliquely incident plane wave in terms of cylindrical waves. Here, we distinguish between two cases: 


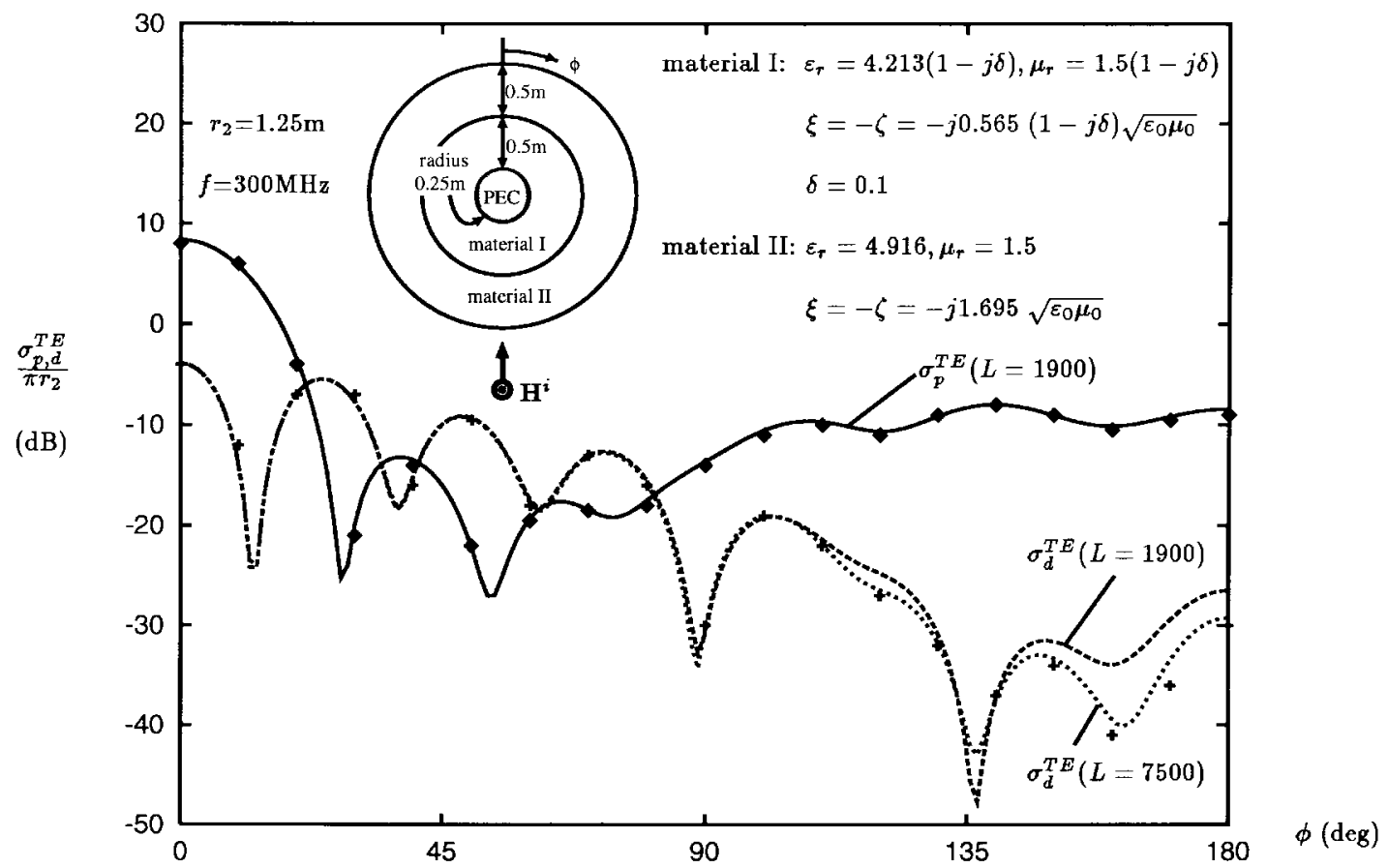

Fig. 2. Polarized and depolarized bistatic echowidths of a two-layer chiral cylinder for an incident TE wave. Comparision of our result (lines) with that of [1, Fig. 3] (points).

1) the incident electric field vector $\mathbf{E}^{i}$ lies in the "incidence plane" defined by the vectors $\mathbf{k}_{0}$ and $\hat{\mathbf{z}}$ (TM case) and 2) the dual case where the incident magnetic field vector $\mathbf{H}^{i}$ lies in that plane (TE case). Hence, we define

$$
\begin{aligned}
\mathbf{E}_{\mathrm{TM}}^{i}=E^{i}\left[-\cos \theta_{0} \cos \left(\phi-\phi_{0}\right) \hat{\mathbf{r}}\right. & \\
& \left.+\cos \theta_{0} \sin \left(\phi-\phi_{0}\right) \hat{\phi}+\sin \theta_{0} \hat{\mathbf{z}}\right] e^{-j \mathbf{k}_{0} \cdot \mathbf{r}}
\end{aligned}
$$

in terms of polar coordinates $\left(E^{i}\right.$ denotes the scalar amplitude, $\hat{\mathbf{r}}, \hat{\phi}, \hat{\mathbf{z}}$ are the unit coordinate vectors), and similarly, for $\mathbf{H}_{\mathrm{TE}}^{i}$. Expanding the exponential as [11]

$$
e^{-j \mathbf{k}_{0} \cdot \mathbf{r}}=\sum_{n=-\infty}^{\infty} j^{-n} J_{n}\left(k_{r} r\right) e^{-j n\left(\phi-\phi_{0}\right)} e^{-j k_{0} \cos \theta_{0} z}
$$

yields, together with Maxwell's equations, the following representation of the TM transverse components in terms of Fourier series

$$
\begin{aligned}
& E_{\phi, \mathrm{TM}}^{i}= E^{i} e^{-j k_{0} \cos \theta_{0} z} \frac{-\cos \theta_{0}}{k_{0} r \sin \theta_{0}} \\
& \times \sum_{n=-\infty}^{\infty} j^{-n} n J_{n}\left(k_{r} r\right) e^{-j n\left(\phi-\phi_{0}\right)} \\
& H_{\phi, \mathrm{TM}}^{i}= E^{i} e^{-j k_{0} \cos \theta_{0} z} \frac{k_{0}}{j \omega \mu_{0}} \\
& \times \sum_{n=-\infty}^{\infty} j^{-n} J_{n}^{\prime}\left(k_{r} r\right) e^{-j n\left(\phi-\phi_{0}\right)} \\
& E_{z, \mathrm{TM}}^{i}= E^{i} e^{-j k_{0} \cos \theta_{0} z} \sin \theta_{0} \sum_{n=-\infty}^{\infty} j^{-n} J_{n}\left(k_{r} r\right) \\
& H_{z, \mathrm{TM}}^{i}=0
\end{aligned}
$$
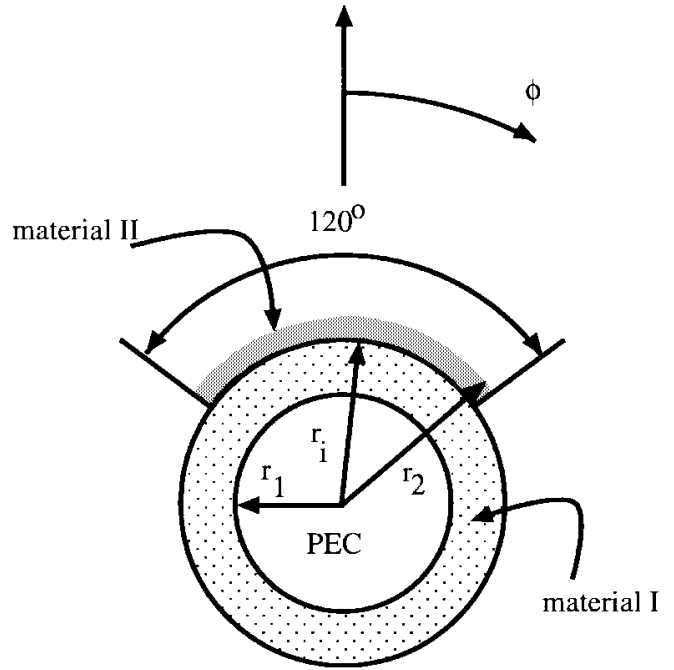

Fig. 3. Cross section corresponding to the results in Figs. 4 and 5 (material data for materials I and II are given in text).

where $J_{n}$ denotes ordinary Bessel's function of order $n$ and the prime indicates derivative with respect to its argument. The expressions for the TE components can be obtained by substituting $E \leftrightarrow H, \mu_{0} \rightarrow-\varepsilon_{0}$ in the above formulas. From these series, the vectors $\tilde{\mathbf{f}}^{i}$ describing the incident field can be readily set up.

To solve for the scattered field, we consider the total field at $r=r_{2}$

$$
\tilde{\mathbf{f}}^{i}\left(r_{2}\right)+\tilde{\mathbf{f}}^{s}\left(r_{2}\right)=\underline{\underline{\mathbf{P}}}\left(r_{2}, r_{1}\right) \cdot \tilde{\mathbf{f}}\left(r_{1}\right)
$$

where we used (7). The subvectors $\left(\overrightarrow{\tilde{E}}_{\phi}^{s}, \overrightarrow{\tilde{E}}_{z}^{s}, \overrightarrow{\widetilde{H}}_{\phi}^{s}\right.$, and $\left.\overrightarrow{\tilde{H}}_{z}^{s}\right)$ of $\tilde{\mathbf{f}}^{s}\left(r_{2}\right)$ are not independent from each other since the $\phi$ components can be expressed in terms of the $z$ components 
as given in (9). Furthermore, an interrelation of the subvectors of $\tilde{\mathbf{f}}\left(r_{1}\right)$ is given by introducing TE/TM surface impedances characterizing the center conductor [12]

$$
Z_{\mathrm{TM}}=\frac{E_{z}\left(r_{1}\right)}{H_{\phi}\left(r_{1}\right)}, \quad Z_{\mathrm{TE}}=-\frac{E_{\phi}\left(r_{1}\right)}{H_{z}\left(r_{1}\right)} .
$$

By considering these relations, the following linear system of equations can be established from (17):

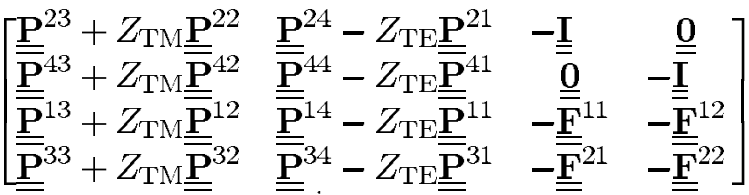

$$
\begin{aligned}
& \cdot\left[\begin{array}{c}
\overrightarrow{\tilde{H}}_{\phi}\left(r_{1}\right) \\
\overrightarrow{\tilde{H}}_{z}\left(r_{1}\right) \\
\overrightarrow{\tilde{E}}_{z}\left(r_{2}\right) \\
\overrightarrow{\tilde{H}}_{z}\left(r_{2}\right)
\end{array}\right]=\left[\begin{array}{c}
\overrightarrow{\tilde{E}}_{z}^{i}\left(r_{2}\right) \\
\overrightarrow{\tilde{H}}_{z}^{i}\left(r_{2}\right) \\
\overrightarrow{\tilde{E}}_{\phi}^{i}\left(r_{2}\right) \\
\overrightarrow{\vec{H}}_{\phi}^{i}\left(r_{2}\right)
\end{array}\right]
\end{aligned}
$$

Here, the matrices $\underline{\mathbf{P}}^{k l l}$ [dimension $\left.(2 N+1) \times(2 N+1)\right]$ are submatrices of the propagator matrix for the inhomogeneous layer

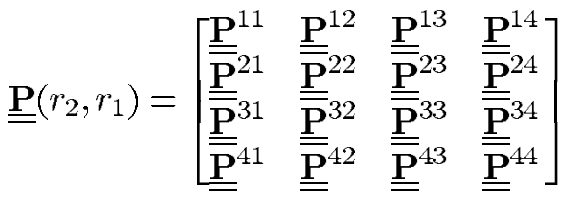

and the matrices $\underline{\mathbf{F}}^{l m}$ represent diagonal matrices being set up by matrix elements $F_{l m, n}$ given in (10); the matrix elements of $\underline{\underline{\mathbf{F}}}^{l m} \mathrm{read}$

$$
F_{i j}^{l m}= \begin{cases}F_{l m, i} ; & i=j \\ 0 ; & i \neq j\end{cases}
$$

Thus, we can solve for the spectral $z$ components of the scattered fields at $r=r_{2}$; the related $\phi$ components then can be easily obtained by (9).

\section{Calculation of the Bistatic Scattering Widths}

In the far field the scattered fields are local plane waves, i.e., $\mathbf{E}^{s}$ and $\mathbf{H}^{s}$ are perpendicular to the direction of propagation, which is described by the wave vector $\mathbf{k}=k_{0}\left(\cos \theta_{0} \hat{\mathbf{z}}+\right.$ $\left.\sin \theta_{0} \hat{\mathbf{r}}\right)$. Hence, the $r$ components in the far field can be expressed in terms of the $z$ components

$$
E_{r}^{s}=-E_{z}^{s} \cot \theta_{0}, \quad H_{r}^{s}=-H_{z}^{s} \cot \theta_{0} .
$$

The bistatic echo width $\sigma$ is defined as [13]

$$
\sigma=2 \pi \lim _{R \rightarrow \infty} R \frac{\mathbf{E}^{s} \cdot \mathbf{E}^{s *}}{E^{i} E^{i *}}=2 \pi \lim _{R \rightarrow \infty} R \frac{\mathbf{H}^{s} \cdot \mathbf{H}^{s *}}{H^{i} H^{i *}}
$$

where $R=r / \sin \theta_{0}$ denotes the far-field distance from the scatterer along the "ray path" of the scattered wave (the asterisk denotes the complex conjugate). Usually, $\sigma$ is divided in a polarized part and a depolarized part. With (22), we have

$\mathbf{E}^{s} \cdot \mathbf{E}^{s *}=\left|E_{z}^{s}\right|^{2}+\left|E_{r}^{s}\right|^{2}+\left|E_{\phi}^{s}\right|^{2}=\left|E_{z}^{s}\right|^{2} / \sin ^{2} \theta_{0}+\left|E_{\phi}^{s}\right|^{2}$ where the contribution $\left|E_{z}^{s}\right|^{2} / \sin ^{2} \theta_{0}$, which represents the $E$-field intensity in the scattering plane accounts for the polarized part, while $\left|E_{\phi}^{s}\right|^{2}$ ( $E$-field intensity perpendicular to the scattering plane) represents the depolarized part for an incident TM-polarized wave [13]. A similar consideration for the scattered magnetic field can be performed for the TE case. By using asymptotic expansions for Hankel functions we can express the scattered far fields in terms of the Fourier coefficients of the transverse fields at $r_{2}$, which leads to the following expressions for the TM bistatic echo widths:

$$
\begin{aligned}
& \sigma_{p}^{\mathrm{TM}}(\phi)=\frac{4}{k_{0} \sin ^{4} \theta_{0}\left|E^{i}\right|^{2}}\left|\sum_{n=-\infty}^{+\infty} \frac{\tilde{E}_{z, n}^{s}\left(r_{2}\right)}{H_{n}^{(2)}\left(k_{r} r_{2}\right)} j^{n} e^{-j n \phi}\right|^{2} \\
& \sigma_{d}^{\mathrm{TM}}(\phi)=\frac{4 \omega^{2} \mu_{0}^{2}}{k_{0}^{3} \sin ^{4} \theta_{0}\left|E^{i}\right|^{2}}\left|\sum_{n=-\infty}^{+\infty} \frac{\tilde{H}_{z, n}^{s}\left(r_{2}\right)}{H_{n}^{(2)}\left(k_{r} r_{2}\right)} j^{n} e^{-j n \phi}\right|^{2}
\end{aligned}
$$

where the subscripts $p$ and $d$ refer to "polarized" and "depolarized," respectively. The corresponding expressions for $\sigma_{p}^{\mathrm{TE}}$ and $\sigma_{d}^{\mathrm{TE}}$ can be obtained by formally substituting $E_{z, n} \leftrightarrow$ $H_{z, n}, E^{i} \rightarrow H^{i}, \mu_{0} \rightarrow \varepsilon_{0}$ in the above formulas.

\section{NUMERICAL RESULTS}

First, the method has been checked by reproducing results for special cases from [13] (perfectly conducting wire covered with homogeneous dielectric layer, normal as well as oblique incidence) and [1] (perfectly conducting wire, homogeneously covered with two layers of lossy chiral material, normal incidence) that yielded very good agreements. For the latter example we show a comparison of results in Fig. 2. Our result was obtained with truncation parameter $N=13$ and $L=1900$ sublayers that account for the considerable electrical thickness of the structure at $300 \mathrm{MHz}$. The results agree very well except for $\sigma_{d}^{\mathrm{TE}}$ in the region below about $-20 \mathrm{~dB}$. However, by increasing the parameter $L$ further, improved agreement can be achieved in this region also (see result for $L=7500$ ).

In the following, we present sample results for a perfectly electrically conducting (PEC) wire (i.e., $Z_{\mathrm{TE}}=Z_{\mathrm{TM}}=0$ ), that is covered by a homogeneous layer and a truncated second layer, which represents a discontinuous inhomogeneity in direction $\phi$. The cross section is shown in Fig. 3, the radii are given by $k_{0} r_{1}=1, k_{0} r_{i}=1.5$, and $k_{0} r_{2}=1.7$, where $k_{0}$ is the free-space wavenumber at the considered frequency.

The inner homogeneous layer is made up of lossy chiral material with $\varepsilon=(3-j 0.003) \varepsilon_{0}, \mu=\mu_{0}, \xi=-\zeta=j 0.6 \sqrt{\varepsilon_{0} \mu_{0}}$ (material I) while the outer, truncated layer consists of uniaxial bianisotropic material with $\underline{\underline{\varepsilon}}=(\underline{\mathbf{I}}-\hat{\mathbf{z}} \hat{\mathbf{z}}) \varepsilon_{t}+\hat{\mathbf{z}} \hat{\mathbf{z}} \varepsilon_{z}$ and accordingly for $\underline{\mu}, \underline{\xi}$, and $\underline{\zeta}$, where $\varepsilon_{t}=5 \varepsilon_{0}, \varepsilon_{z}=6 \varepsilon_{0}, \mu_{t}=$ $3 \mu_{0}, \mu_{z}=4 \mu_{0}, \bar{\xi}_{t}=-\zeta_{t}=-j 0.8 \sqrt{\varepsilon_{0} \mu_{0}}$ and $\xi_{z}=-\zeta_{z}=$ $-j 0.9 \sqrt{\varepsilon_{0} \mu_{0}}$ (material II). In the following, we will compare results for these layer materials (labeled $b i$ for short) and corresponding "ordinary" materials (labeled non-bi) which are obtained by setting the $\xi$ and $\zeta$ parameters for material I and II to zero. 

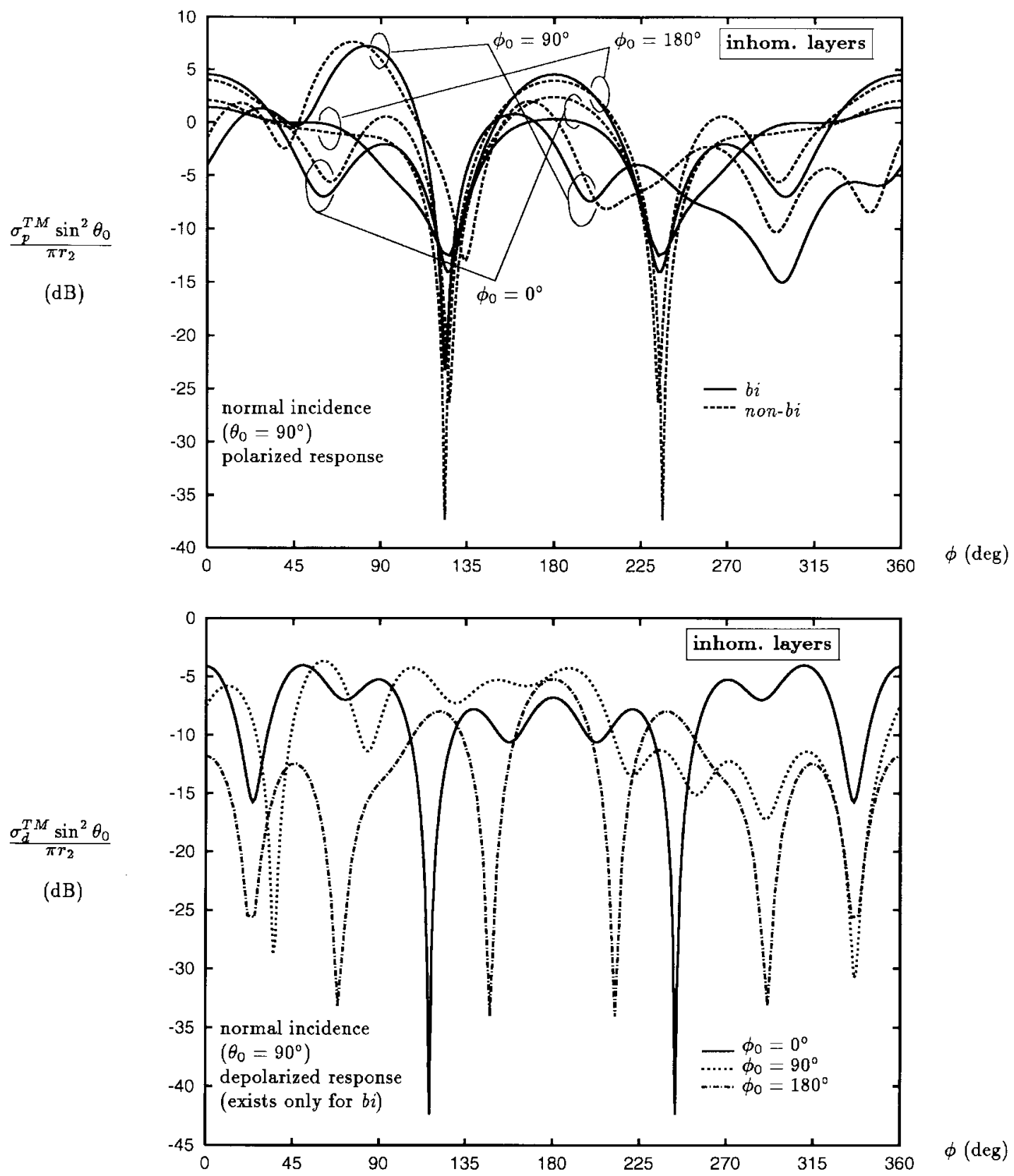

Fig. 4. Bistatic echo widths for normal TM incidence; polarized and depolarized parts for the $b i$ and non-bi case (no depolarization occurs in the later case).

In the calculations, it was found that $L=200$ sublayers and $N=10$ for the truncation parameter (corresponding to $2 N+1=21$ considered space harmonics) ensured numerical convergence. We consider scattering of a TM wave for normal incidence $\left(\theta_{0}=90^{\circ}\right.$, Fig. 4) and oblique incidence with $\theta_{0}=$ $18^{\circ}$ (Fig. 5).

Due to the angular inhomogeneity of the structure, the scattering behavior depends on the incidence angle $\phi_{0}$. In our calculations, three angles $\phi_{0}$ are assumed: $\phi_{0}=0^{\circ}, 90^{\circ}$, and $180^{\circ}$. For normal incidence, the curves for the cases $\phi_{0}=0^{\circ}$ and $180^{\circ}$ are symmetric with respect to $\phi=0^{\circ}$ and $180^{\circ}$, while no corresponding symmetry can be observed for the case $\phi_{0}=90^{\circ}$. Generally, a sufficient condition for such a symmetry is that the structure under consideration transforms into itself after performing a $180^{\circ}$ rotation around the direction of the incident wave. Note that this rotation affects not only geometrical boundaries but generally also (bi)anisotropic material tensors. In our case, however, the bianisotropy is uniaxial with respect to $z$, which is not affected by $180^{\circ}$ rotations around axes perpendicular to $z$. Hence, the lack of symmetry in the case $\phi_{0}=90^{\circ}$ is essentially caused by the truncated top layer.

In the case of oblique incidence, no such symmetry is observed for any of the three incidence angles $\phi_{0}$ for the bi case. However, for $\phi_{0}=0^{\circ}$ and $180^{\circ}$ symmetries occur for the non-bi case (see Fig. 5). Hence, the lack of such symmetries in the case of oblique incidence is essentially caused by the presence of bianisotropic media. A derivation of sufficient conditions for these symmetries is discussed in Appendix B. 

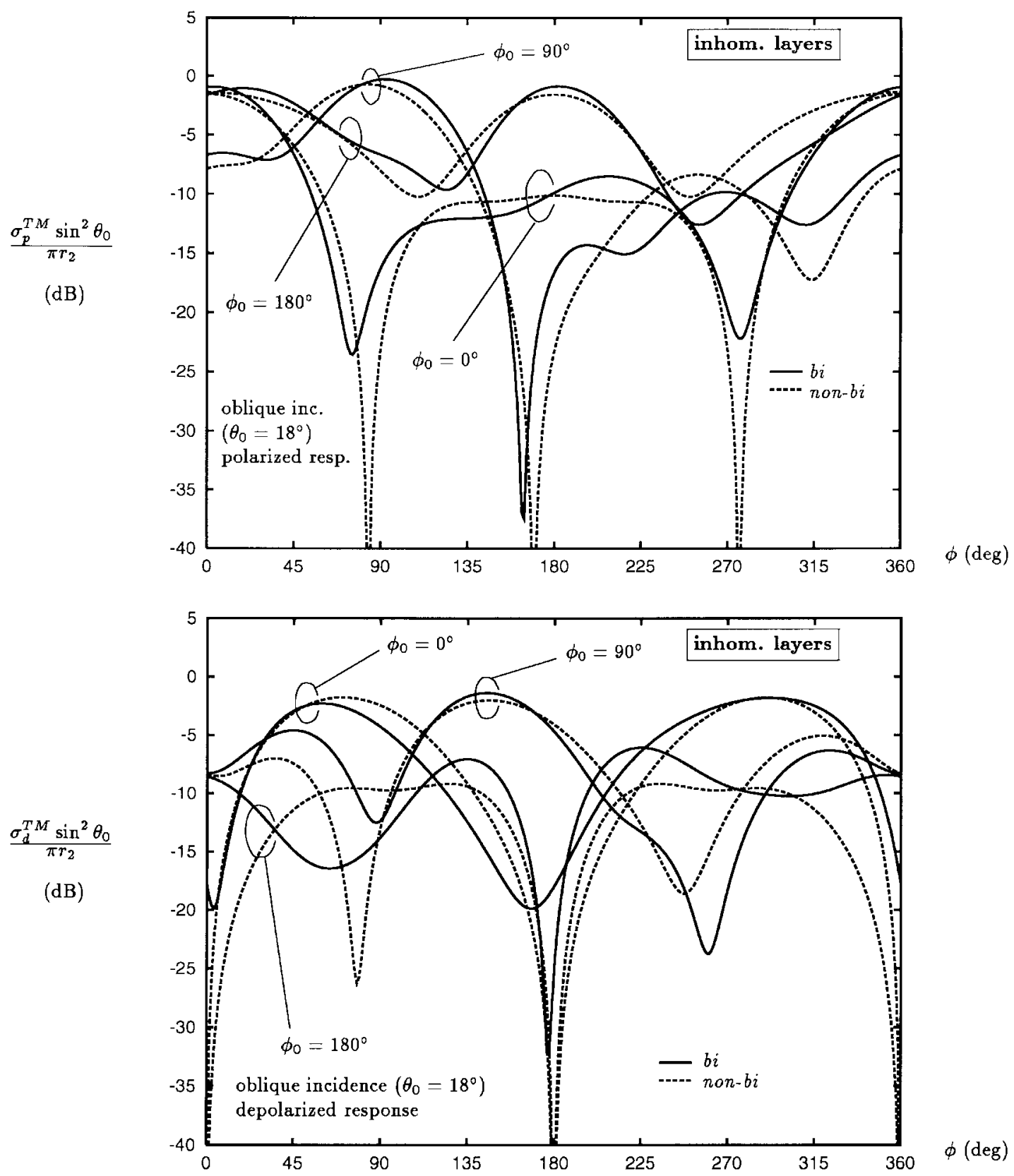

Fig. 5. Bistatic echo widths for normal TM incidence $\left(\theta_{0}=18^{\circ}\right)$; polarized and depolarized parts for the bi and non-bi case.

Apart from introducing asymmetry in the scattering width plots, we further note that the bianisotropic character of the media introduces significant depolarized contributions even for normal incidence (in contrast to the non-bi case, which yields no depolarized fields for normal incidence, see Fig. 4). This has also been found for chiral (reciprocal bi-isotropic) media (see, e.g., [1] and Fig. 2). In the case of oblique incidence, we have depolarized scattered fields for the $b i$ as well as for the non-bi case, however, the curves are quite different in shape in some regions (see, e.g., the curves for $\phi_{0}=180^{\circ}$ in the region around $\phi=0^{\circ}$ ). This, again, indicates the influence of the "bi" behavior on the depolarized scattered fields.

The chosen example of a partial coating represents a simple, practically realizable, geometry featuring a layer with angular inhomogeneity. Of course, problems with continuous varia- tions of the material parameters with the coordinate $\phi$ can be treated in exactly the same manner. However, since we are dealing with a Fourier method with respect to to the angular coordinate $\phi$, the case of discontinuous material parameters represents a good numerical test case, since, compared to an example with continuous parameters, a high number of spatial harmonics has to be expected to obtain numerical convergence.

\section{SUMMARY}

In this contribution an approach for the solution of scattering problems involving inhomogeneous cylindrical structures has been presented. The method is based upon the numerical calculation of the propagator matrix for an inhomogeneous layer which is performed in the spectral domain where field quantities as well as material parameter functions are expanded 
in Fourier series with respect to $\phi$. This yields an infinite dimensional propagator matrix, which is truncated for numerical calculation. The latter is then used to set up a linear system of equations for the Fourier coefficients of scattered fields due to an obliquely incident plane wave. The method has been checked by comparison with previously obtained results for cylindrical structures involving homogeneous layers of (bi)isotropic media, which are included as special cases in our method. A sample result has illustrated a typical application of the method and depolarization, and asymmetries in the scattered field response due to the presence of bianisotropic media have been discussed.

\section{APPENDIX A}

\section{THE MATRIX OPERATOR $\underline{\underline{\mathcal{L}}}$}

Considering the material parameter tensors in cylindrical coordinates $(r, \phi, z)$, the $4 \times 4$ matrix operator $\underline{\underline{\mathcal{L}}}$ in (3) is given by

$$
\begin{aligned}
& \mathcal{L}_{11}=-\zeta_{z \phi}-\frac{1}{j \omega r}+\left(\partial_{\phi}-\zeta_{z r}\right) A-\mu_{z r} B \\
& \mathcal{L}_{21}=\zeta_{\phi \phi}+\left(\partial_{z}+\zeta_{\phi r}\right) A+\mu_{\phi r} B \\
& \mathcal{L}_{31}=\varepsilon_{z \phi}+\varepsilon_{z r} A+\left(\partial_{\phi}+\xi_{z r}\right) B \\
& \mathcal{L}_{41}=-\varepsilon_{\phi \phi}-\varepsilon_{\phi r} A+\left(\partial_{z}-\xi_{\phi r}\right) B \\
& \mathcal{L}_{12}=-\zeta_{z z}+\left(\partial_{\phi}-\zeta_{z r}\right) C-\mu_{z r} D \\
& \mathcal{L}_{22}=\zeta_{\phi z}+\left(\partial_{z}+\zeta_{\phi r}\right) C+\mu_{\phi r} d \\
& \mathcal{L}_{32}=\varepsilon_{z z}+\varepsilon_{z r} C+\left(\partial_{\phi}+\xi_{z r}\right) D \\
& \mathcal{L}_{42}=-\varepsilon_{\phi z}-\varepsilon_{\phi r} C+\left(\partial_{z}-\xi_{\phi r}\right) D \\
& \mathcal{L}_{13}=-\mu_{z \phi}+\left(\partial_{\phi}-\zeta_{z r}\right) E-\mu_{z r} F \\
& \mathcal{L}_{23}=\mu_{\phi \phi}+\left(\partial_{z}+\zeta_{\phi r}\right) E+\mu_{\phi r} F \\
& \mathcal{L}_{33}=\xi_{z \phi}-\frac{1}{j \omega r}+\varepsilon_{z r} E+\left(\partial_{\phi}+\xi_{z r}\right) F \\
& \mathcal{L}_{43}=-\xi_{\phi \phi}-\varepsilon_{\phi r} E+\left(\partial_{z}-\xi_{\phi r}\right) F \\
& \mathcal{L}_{14}=-\mu_{z z}+\left(\partial_{\phi}-\zeta_{z r}\right) G-\mu_{z r} H \\
& \mathcal{L}_{24}=\mu_{\phi z}+\left(\partial_{z}+\zeta_{\phi r}\right) G+\mu_{\phi r} H \\
& \mathcal{L}_{34}=\xi_{z z}+\varepsilon_{z r} G+\left(\partial_{\phi}+\xi_{z r}\right) H \\
& \mathcal{L}_{44}=-\xi_{\phi z}-\varepsilon_{\phi r} G+\left(\partial_{z}-\xi_{\phi r}\right) H
\end{aligned}
$$

where

$$
\begin{aligned}
& A=-\frac{\varepsilon_{r \phi} \mu_{r r}}{d_{r}}-\frac{\xi_{r r}\left(\partial_{z}-\zeta_{r \phi}\right)}{d_{r}} \\
& B=\frac{\varepsilon_{r \phi} \zeta_{r r}}{d_{r}}+\frac{\varepsilon_{r r}\left(\partial_{z}-\zeta_{r \phi}\right)}{d_{r}} \\
& C=-\frac{\varepsilon_{r z} \mu_{r r}}{d_{r}}-\frac{\xi_{r r}\left(-\partial_{\phi}-\zeta_{r z}\right)}{d_{r}} \\
& D=\frac{\varepsilon_{r z} \zeta_{r r}}{d_{r}}+\frac{\varepsilon_{r r}\left(-\partial_{\phi}-\zeta_{r z}\right)}{d_{r}} \\
& E=\frac{\mu_{r \phi} \xi_{r r}}{d_{r}}+\frac{\mu_{r r}\left(-\partial_{z}-\xi_{r \phi}\right)}{d_{r}} \\
& F=-\frac{\varepsilon_{r r} \mu_{r \phi}}{d_{r}}-\frac{\zeta_{r r}\left(-\partial_{z}-\xi_{r \phi}\right)}{d_{r}} \\
& G=\frac{\mu_{r z} \xi_{r r}}{d_{r}}+\frac{\mu_{r r}\left(\partial_{\phi}-\xi_{r z}\right)}{d_{r}}
\end{aligned}
$$

$$
H=-\frac{\varepsilon_{r r} \mu_{r z}}{d_{r}}-\frac{\zeta_{r r}\left(\partial_{\phi}-\xi_{r z}\right)}{d_{r}}
$$

and

$$
\begin{gathered}
d_{r}=\varepsilon_{r r} \mu_{r r}-\xi_{r r} \zeta_{r r} \\
\partial_{\phi}=\frac{1}{j \omega r} \frac{\partial}{\partial \phi}, \quad \partial_{z}=\frac{1}{j \omega} \frac{\partial}{\partial z} .
\end{gathered}
$$

Note that operators occurring in the numerator of fractions do not act on the denominator $d_{r}$.

\section{APPENDIX B \\ SYMMETRIES IN $\sigma_{p, d}(\phi)$}

To discuss the appearance of symmetries in the $\sigma_{p, d}(\phi)$ characteristics a bit more in detail, let us briefly investigate how the sufficient conditions mentioned in Section III can be derived. For the following considerations, a rectangular coordinate frame $x, y, z$ is best suited.

Let us first discuss the case of normal incidence. Assume that a plane wave traveling in $x$ direction is incident on a structure characterized by material parameter tensors $\underline{\underline{\varepsilon}}(x, y)$, $\underline{\mu}(x, y), \underline{\xi}(x, y)$, and $\underline{\zeta}(x, y)$ (we consider no inhomogeneity with respect to $z$ ). Assume, further, that the solution of this scattering problem is known, and let us denote the solution functions for the field variables by small letters, i.e.,

$$
E_{\alpha}=e_{\alpha}(x, y, z), \quad H_{\alpha}=h_{\alpha}(x, y, z)
$$

with $\alpha=x, y$, and $z$. Next, we introduce new independent variables $y^{\prime}=-y$ and $z^{\prime}=-z$ into the source-free Maxwell's equations, but keep considering the field components in the frame $x, y, z$. If our geometry is composed of biaxial (diagonally) bianisotropic media that fulfill the symmetry properties

$$
\eta_{i i}(x, y)=\eta_{i i}(x,-y)
$$

where $i=x, y, z$ and $\eta$ stands for $\varepsilon, \mu, \xi$, and $\zeta$, it can easily be shown that the set

$$
\begin{gathered}
E_{x}=-e_{x}\left(x, y^{\prime}, z^{\prime}\right)=-e_{x}(x,-y,-z) \\
H_{x}=-h_{x}\left(x, y^{\prime}, z^{\prime}\right)=-h_{x}(x,-y,-z) \\
E_{\nu}=e_{\nu}\left(x, y^{\prime}, z^{\prime}\right)=e_{\nu}(x,-y,-z) \\
H_{\nu}=h_{\nu}\left(x, y^{\prime}, z^{\prime}\right)=h_{\nu}(x,-y,-z)
\end{gathered}
$$

(with $\nu=y, z$ ) formally represents a solution of the sourcefree field equations as well. Our incident wave features no $x$ component and constant $y, z$ components in $x=$ const planes, and is thus compatible with both solutions (53) and (55). Assuming that the corresponding solution for the scattered fields is unique, the solution for the scattered field must also be consistent with (53) and (55), and we thus obtain the following symmetry conditions for the field components:

$$
\begin{gathered}
E_{x}(x, y, z)=-E_{x}(x,-y,-z) \\
H_{x}(x, y, z)=-H_{x}(x,-y,-z) \\
E_{\nu}(x, y, z)=E_{\nu}(x,-y,-z) \\
H_{\nu}(x, y, z)=H_{\nu}(x,-y,-z)
\end{gathered}
$$


where $\nu=y, z$. This means that the $x$ axis represents a mirror axis for the Poynting vector $\mathbf{S}=\mathbf{E} \times \mathbf{H}^{*}$. Since we have no field variation in the $z$ direction, (56a) and (56b) means that the scattered power in some direction $\phi$ is the same as in direction $-\phi$, where $\phi$ denotes the angle with respect to the $x$ axis (azimuth). This explains the symmetries for $\phi_{0}=0^{\circ}$ and $180^{\circ}$ in the normal incidence case observed in Fig. 4 for the $b i$ as well as the non-bi case.

In the case of oblique incidence, the above approach fails since the incident wave is not compatible with (53) and (55). Alternatively we can tackle the problem as follows. Let us assume that the incident wave vector lies in the $x, z$ plane and consider the TM case, where the incident electric field vector lies in the $x, z$ plane as well (orthogonal to the direction of propagation, of course). For the TE case dual considerations apply. If we again assume that the solution (53) of the problem is known and introduce a new independent variable $y^{\prime}=-y$, one eventually finds in a similar manner as above that the set

$$
\begin{aligned}
& E_{x}=e_{x}(x,-y, z), \\
& E_{y}=-e_{y}(x,-y, z) \\
& E_{z}=e_{z}(x,-y, z) \\
& H_{x}=-h_{x}(x,-y, z) \\
& H_{y}=h_{y}(x,-y, z) \\
& H_{z}=-h_{z}(x,-y, z)
\end{aligned}
$$

is also a solution of the source-free Maxwell's equations, provided that 1) the geometry consists of biaxial "ordinary" anisotropic media and 2) the material parameter tensors fulfill (54) with $\eta=\varepsilon, \mu$. It is important to note that this approach fails for bi-media. Similarly, as above, the incident plane wave is compatible with (57) and (53), which means that the scattered fields also have to be consistent with (57) and (53). This means that the $x, z$ plane is a mirror plane for the Poynting vector and, thus, the scattered power is the same in directions characterized by azimuthal angles $\phi$ and $-\phi$. Of course, this approach can also be used to show the symmetry properties for the normal incidence case, which is a special case of the oblique incidence case, but since it works for "ordinary" media only, we do not obtain any conclusions for the $b i$ case.

Again, we note that both considerations yield sufficient conditions, only. However, in our numerical sample calculations, the symmetry properties are violated in all the cases not covered by the sufficient conditions derived above.

\section{ACKNOWLEDGMENT}

The author would like to thank F. Olyslager and D. De Zutter of the University of Ghent, Belgium, for valuable discussions, and Prof. F. Seifert, Vienna University of Technology, Austria, for his continuous encouragement.

\section{REFERENCES}

[1] M. S. Kluskens and E. H. Newman, "Scattering by a multilayer chiral cylinder," IEEE Trans. Antennas Propagat., vol. 39, pp. 91-96, Jan. 1991.

[2] K. Chittayil and A. Lakhtakia, "Electromagnetic scattering by a chiral cylinder immersed in another chiral medium," Optik, vol. 89, pp. 59-64, Dec. 1991.

[3] R. D. Graglia, P. L. E. Uslenghi, and C. L. Yu, "Electromagnetic oblique scattering by a cylinder coated with chiral layers and anisotropic jumpimmittance sheets," J. Electromagn. Waves Applicat., vol. 6, no. 5/6, pp. 695-719, 1992.

[4] A. K. Bhattacharyya and A. Lakhtakia, "On the scattering of an obliquely incident plane wave by a bi-isotropic cylinder," Int. J. Infrared Millimeter Waves, vol. 13, pp. 995-1005, July 1992.

[5] Z.-X. Shen, "Electromagnetic scattering by an impedance cylinder coated eccentrically with a chiroplasma cylinder," in Proc. Inst. Elect. Eng. Microwave Antennas Propagat., vol. 141, pp. 279-285, Aug. 1994.

[6] D. L. Jaggard and J. C. Liu, "Chiral layers on curved surfaces," $J$. Electromagn. Waves Applicat., vol. 6, no. 5/6, pp. 669-694, 1992.

[7] I. V. Lindell, A. H. Sihvola, S. A. Tretyakov, and A. J. Viitanen, Electromagnetic Waves in Chiral and Bi-Isotropic Media. Boston, MA: Artech, 1994.

[8] A. Lakhtakia, V. Varadan, and V. Varadan, Time Harmonic Electromagnetic Fields in Chiral Media. New York: Springer-Verlag, 1989 vol. 335, lecture notes phys.

[9] B. Jakoby and D. De Zutter, "Analysis of guided waves in inhomogeneous bianisotropic cylindrical waveguides," Trans. Microwave Theory Tech., vol. 44, pp. 297-310, Feb. 1996.

[10] J. A. Kong, Electromagnetic Wave Theory, 2nd ed. New York: Wiley, 1990.

[11] J. A. Stratton, Electromagnetic Theory. New York: McGraw-Hill, 1941.

[12] T. B. A. Senior, "Approximate boundary conditions," IEEE Trans. Antennas Propagat., vol. AP-29, pp. 826-829, Sept. 1981.

[13] G. T. Ruck, D. E. Barrick, W. D. Stuart, and C. K. Krichbaum, Radar Cross Section Handbook. New York: Plenum, vol. 1, 1970.

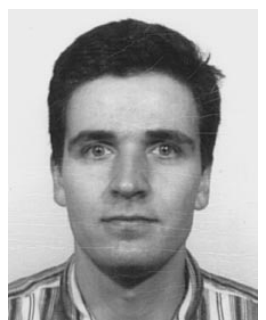

Bernhard Jakoby (S'90-M'94) was born on November 17, 1966, in Neuß, Germany. He received the Dipl.-Ing. and Ph.D. degrees from the Vienna University of Technology, Austria, in 1991 and 1994, respectively.

From 1989 to 1991, he worked as a Tutor (Teaching Assistant) at the Institute of Communication- and Radio-Frequency Engineering, Vienna University of Technology, Austria, and from 1991 to 1994 as a Research Assistant at the Institute of Electronics (IAEE) at the same university. From August 1994 to September 1995 he was a Guest Scientist at the Department of Information Technology, University of Ghent, Belgium, through an Erwin Schrödinger Grant from the Austrian Fund for Scientific Research (FWF). Currently, he is with the Electronic Instrumentation Department, Delft University of Technology, The Netherlands, where he is working on the design and realization of microacoustic sensors. His research interests are focused on numerical and analytical methods, complex media in electromagnetics and acoustics, and microacoustics, in general. 